\title{
CONF- $970711--15$ \\ RADIATION-INDUCED GAIN DEGRADATION IN LATERAL PNP BJTS WITH LIGHTLY AND HEAVILY DOPED EMITTERS
}

\author{
A.Wu ${ }^{1}$, R.D. Schrimp ${ }^{2}$, R.L. Pease ${ }^{3}$, D.M Fleetwood ${ }^{4}$, and S.L. Kosier ${ }^{5}$ \\ 1. Department of ECE, University of Arizona, Tucson, AZ 85721 \\ 2. Department of ECE, Vanderbilt University, Nashville, TN 37235 \\ 3. RLP Research, Inc., Albuquerque, NM 87122 \\ 4. Sandia National Laboratories, Albuquerque, NM 87185-1083 \\ 5. VTC Inc., Bloomington, MN 55425-1350
}

\section{Abstract}

Radiation induced gain degradation is compared as a function of dose rate and emitter doping. Devices with lightly doped emitters degrade more rapidly with dose, but the damage mechanisms are otherwise similar.

\section{DISCLAIMER}

This report was prepared as an account of work sponsored by an agency of the United States Government. Neither the United States Government nor any agency thereof, nor any of their employees, makes any warranty, express or implied, or assumes any legal liability or responsibility for the accuracy, completeness, or usefulness of any information, apparatus, product, or process disclosed, or represents that its use would not infringe privately owned rights. Reference herein to any specific commercial product, process, or service by trade name, trademark, manufacturer, or otherwise does not necessarily constitute or imply its endorsement, recommendation, or favoring by the United States Government or any agency thereof. The views and opinions of authors expressed herein do not necessarily state or reflect those of the United States Government or any agency thereof.

\section{ACKNOWLEDGMENTS}

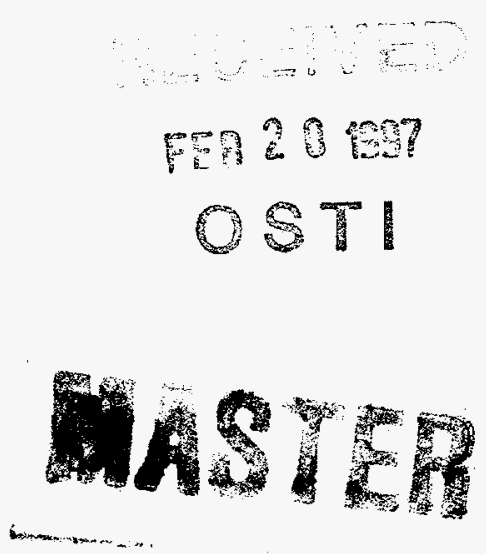

This work was supported by DNA and Intel. The authors wish to thank VTC Inc. for their support in providing process information and devices. The technical support and useful technical discussions of Peter Hopper, Dawn Schmidt and Mark Allenspach were especially helpful during this project. Harry Doane and Wayne Lohmeier of the University of Arizona are greatly appreciated for their experimental assistance. In addition, the authors are grateful to Lew Cohn of DNA and Ken Galloway at Vanderbilt University for their interest in this work. Sandia is a multiprogram laboratory operated by Sandia Corporation, a Lockheed Martin Company, for the US Department of Energy under Contract DE-AC04-94AL85000. 


\section{DISCLAMMER}

Portions of this document may be illegible in electronic image products. Images are produced from the best available original document. 


\section{INTRODUCTION}

Ionizing radiation may cause failures in ICs due to gain degradation of individual devices. The base current of irradiated bipolar devices increases with total dose, while the collector current remains relatively constant. This results in a decrease in the current gain $\left(\beta=I_{C} \pi_{B}\right)$. Lateral PNP (LPNP) transistors typically exhibit more degradation than vertical PNP devices at the same total dose, and have been blamed as the cause of early IC failures at low dose rates [1-4].

Radiation-induced changes in bipolar device characteristics are caused by generation of net positive oxide trapped charge, and an increase in surface recombination velocity due to formation of interface traps. The effect of the radiation-induced damage is most severe at the surface of the device. This leads to increased degradation in the LPNP devices, where the current travels along the surface near the $\mathrm{Si} / \mathrm{SiO}_{2}$ interface. The oxide charge causes an accurmulation layer to form below the $\mathrm{Si} / \mathrm{SiO}_{2}$ interface in the n-type base, whereas a depletion region forms below the interface of the p-type emitter. Surface recombination increases in depleted regions and is reduced in accumulated regions. In LPNP devices with heavily-doped emitters, surface depletion in the emitter is slight and degradation is due almost entirely to increased surface recombination in the base.

LPNP devices previously studied in detail $[1,5,6]$ had emitter doping in the neighborhood of $1 \times 10^{20} \mathrm{~cm}^{-3}$, but devices currently being used in most space applications have more lightly-doped emitters. The degradation of the devices of the RF25 process of Analog Devices was examined previously using analytical modeling and two-dimensional simulations [1]. Schmidt et al. showed that the interface traps introduced at the $\mathrm{Si} / \mathrm{SiO}_{2}$ interface resulted in an increase in base current. However, the net positive charge in the oxide moderates this increase by accumulating the base and reducing surface recombination. In general, the positive charge also causes the emitter depletion region to extend into the p-type emitter. This causes increased surface recombination at the oxide interface over the emitter, as illustrated schematically in Fig. 1.

This is the same effect that occurs in the base region of irradiated NPN devices [7-11]. The amount of this depletion

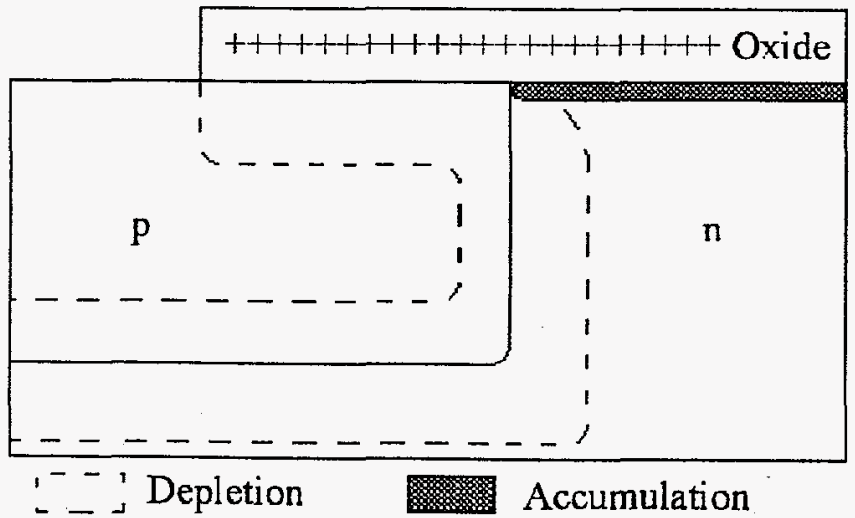

Figure 1. Cross-section of the emitter base junction illustrating depletion and accumulation regions in the presence of positive oxide charge depends on emitter doping; for heavily doped emitters the increase in depletion-region width is virtually nonexistent. On the other hand the lightly doped structures have a substantial extension of the depletion region into the emitter.

It is important to understand the differences in total-dose response between devices with heavily- and lightly-doped emitters in order to compare different technologies and evaluate the applicability of proposed low-dose-rate hardness-assurance methods. This paper addresses these differences by comparing two different LPNP devices from the same process: one with a . heavily-doped emitter and one with a lightly-doped emitter. Experimental results demonstrate that the lightly-doped devices are more sensitive to ionizing radiation and simulations illustrate that increased recombination on the emitter side of the junction is responsible for the higher sensitivity.

\section{EXPERIMENTS AND SIMULATIONS}

The two LPNP devices studied in this work were from the PolarMOS3 process from VTC, which is a Complementary BiCMOS process. The emitter and collector of the heavilydoped-emitter LPNP transistors are created from the same implant and diffusions used to form the source/drain regions of the accompanying MOSFETS. The emitter and collector of the lightly-doped LPNP transistors are formed from the base diffusion of an NPN device in the process. The two devices are otherwise identical as illustrated in Fig. 2.

The heavily-doped emitter structure was doped at $10^{20} \mathrm{~cm}^{-3}(\mathrm{P}+$ LPNP) and the lightly doped emitter was doped at $10^{18} \mathrm{~cm}^{-3}(\mathrm{P}$ LPNP). Both devices are created on top of the same foundation where the base doping is $3 \times 10^{16} \mathrm{~cm}^{-3}$. The devices are cylindrically symmetric about the emitter.

The emitter diameter is $2.4 \mu \mathrm{m}$ with a junction depth of $0.45 \mu \mathrm{m}$. The oxide thickness over the neutral base is $400 \mathrm{~nm}$ with a neutral base width of $2.6 \mu \mathrm{m}$. The "channel" between the emitter and the collector is due to the threshold-adjust implant for the MOSFETS. This implant is fully depleted at zero bias conditions and becomes inverted as the emitter-base junction is forward biased, creating an n-type base. The extended field plate for the emitter contact helps invert the surface of the neutral base. No effect of this implant on the device electrical characteristics was seen in the simulations described here.

The devices were irradiated in a Co-60 source at the University of Arizona at $1 \mathrm{rad}(\mathrm{Si}) / \mathrm{s}, 0.1 \mathrm{rad}(\mathrm{Si}) / \mathrm{s}, 0.05 \mathrm{rad}(\mathrm{Si}) / \mathrm{s}$, and $0.01 \mathrm{rad}(\mathrm{Si}) / \mathrm{s}$. The devices had all pins grounded during irradiation and were exposed to a total dose of 200

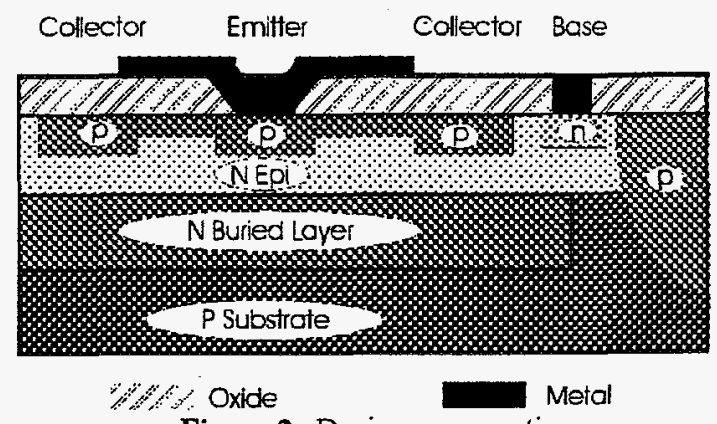

Figure 2. Device cross-section 
krad(Si). The Gummel plots for the devices were obtained with the substrate at $-3 \mathrm{~V}$, the collector at $-2.5 \mathrm{~V}$, the base grounded, and the emitter swept from $0 \mathrm{~V}$ to $1 \mathrm{~V}$.

Simulated device current-voltage characteristics were obtained using ATLAS from SILVACO. In the simulations, net oxide charge $\left(N_{o x}\right)$ and surface recombination velocity (SRV) were varied in a systematic matrix with $N_{o x}$ ranging from 0 to $5 \times 10^{12} \mathrm{~cm}^{-2}$ and SRV ranging from 0 to $10^{6} \mathrm{~cm} / \mathrm{s}$.

\section{RESULTS}

The current gain is plotted vs. emitter-base voltage $\left(V_{\mathrm{EB}}\right)$ in Fig. 3 for both device types. The devices with the lightly-doped emitters started with lower gains and also suffered more degradation with total dose. The lower initial gain was due to increased back injection from the base to the emitter. The reason for the enhanced degradation is explored in more detail below. Both device types examined here showed enhanced degradation at the lowest dose rates, similar to the devices studied earlier from the Analog Devices RF25 process [7]. The increase in $\Delta(1 / \beta)$ at the lowest dose rate examined here is approximately 2 times greater then at the highest dose rate for both device types. This dose-rate dependence will be discussed in more detail in the full paper.

The radiation-induced gain degradation was due primarily to increased base current, as illustrated in Fig. 4. The P- LPNP devices initially had higher base current than the P+ LPNP devices, because the low emitter doping results in more-back Heavily Daped Emitter

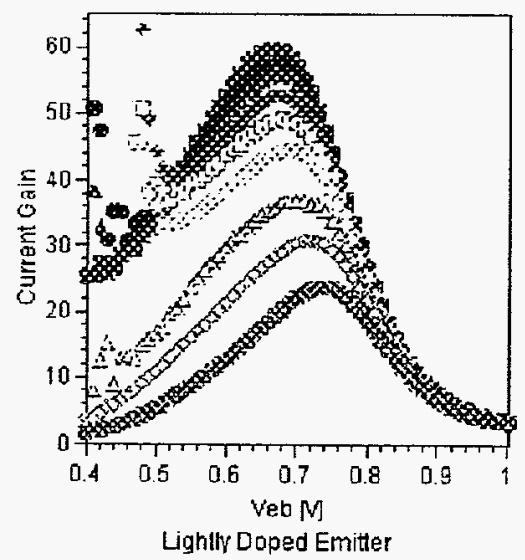

$$
\begin{aligned}
& \text { \& Prerad } \\
& \text { 1k Total Dose } \\
& \& \text { 2k Tokal Dose } \\
& \text { 5k Total Dose } \\
& \therefore \quad \text { 10k Total Dose } \\
& \therefore \text { 50k Total Dose } \\
& \& \text { 100k Total Dose } \\
& * 200 k \text { Total Dose }
\end{aligned}
$$

Lighilly Doped Emitter

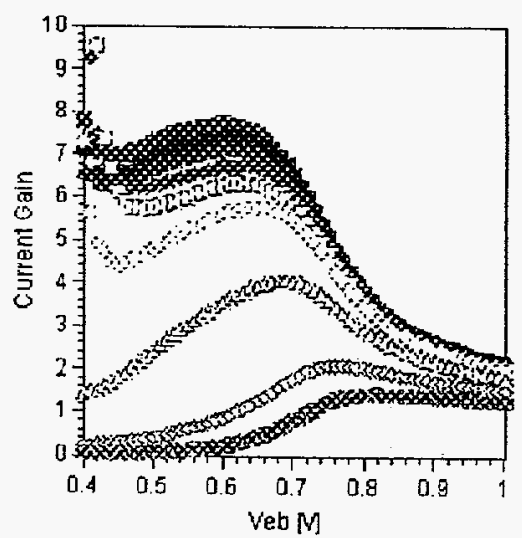

Figure 3. Experimental current gain comparison between the two VTC parts at a dose rate of $0.1 \mathrm{rad} / \mathrm{s}$

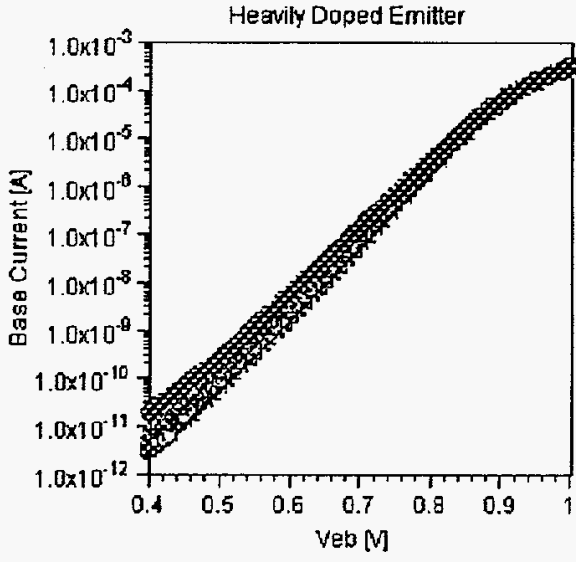

$$
\begin{array}{|ll}
\hline \text { I } & \text { IE (Prorad) } \\
\text { C } & \text { 旧 (50k) } \\
\therefore & \text { IE (100k) } \\
\text { - } & \text { IE (200k) }
\end{array}
$$

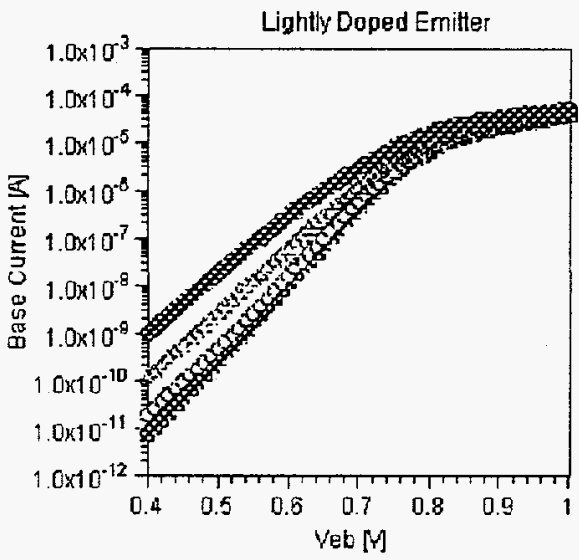

Figure 4. Experimental base current comparison

injected carriers from the base. In addition to the increased base current, there was a slight decrease in collector current with total dose. The collector currents in both device types were initially similar, aside from a slight bend-over in the P- LPNP devices at high biases due to high-level effects. The decrease in collector current due to irradiation was identical in both devices, because of the similar base doping levels. This decrease of collector current is due to an increase in the recombination of carriers along the surface of the device, mainly in the neutral base.

The mechanisms responsible for the gain degradation in both device types were examined using two-dimensional simulations. The surface recombination velocity and positive oxide trapped charge were varied in order to accurately match the pre-irradiation experimental Gummel plots. Figure 5 shows that the simulated device characteristics closely match the experimental characteristics when $N_{o x}=5 \times 10^{10} \mathrm{~cm}^{-2}$ and SRV $=0$ $\mathrm{cm} / \mathrm{s}$, which are physically realistic values for this technology. In Fig. 5, the filled symbols represent the simulated characteristics, and the empty symbols represent the experimental pre-irradiation data. The agreement is excellent except at high bias levels in the P- LPNP device, which shows a bend-over due to high contact resistance in the lightly-doped emitter and collector. 

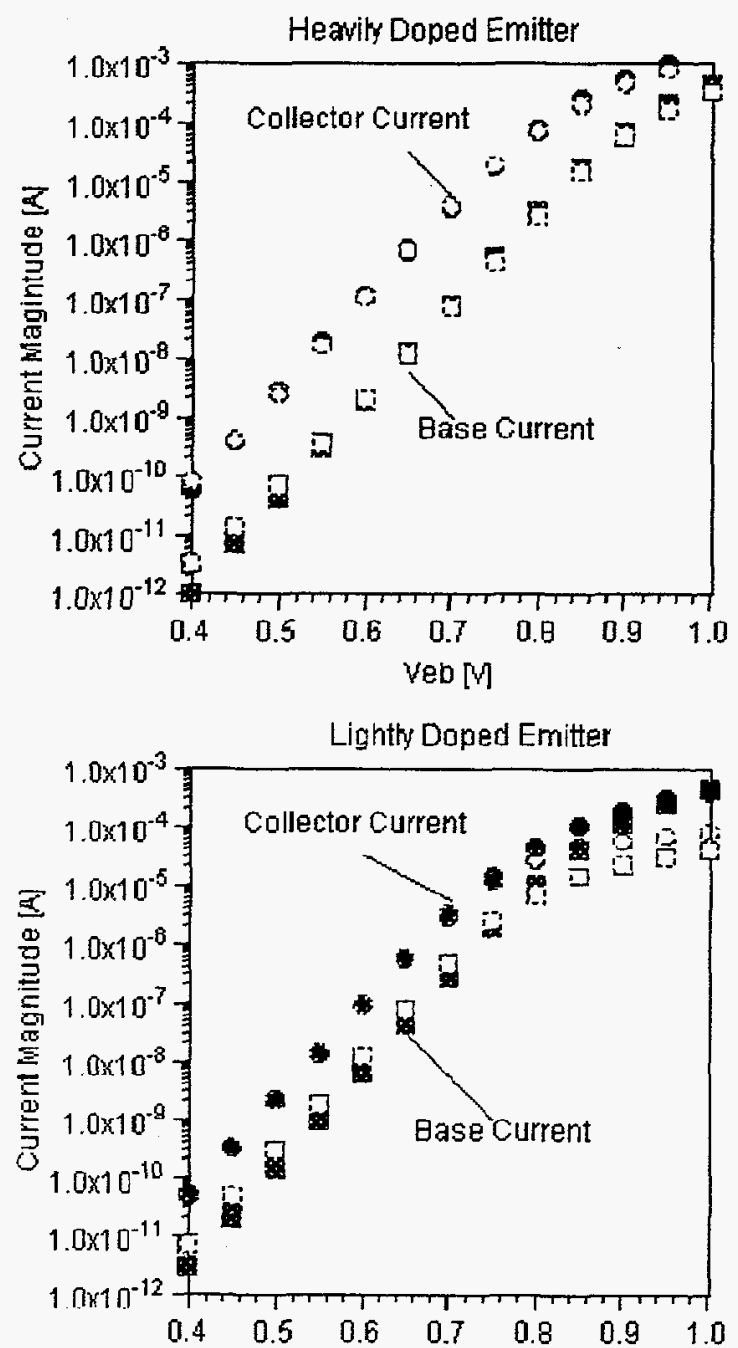

Figure 5. Comparison of simulated and experimental Gurnmel sweeps

The simulations were used to study the relative effects of net oxide charge and surface recombination velocity in the two device types. Figures 6 and 7 show that increasing surface recombination velocity increases the magnitude of the base current, while increasing the net oxide charge changes the slope of the $I_{B}-$ vs. $-V_{E S}$ curve. In the base region, these two mechanisms oppose each other, but in both device types, the dominating effect is the increase in surface recombination velocity. In the devices with lightly-doped emitters, there is a larger increase in base current with increasing surface recombination velocity. This is due to the larger extent of the emitter/base depletion region on the emitter side of the junction.

Increased positive oxide charge reduces the excess base current (for a given surface recombination velocity) in both device types. However, the benefit is less in the lightly-doped devices because, in addition to accumulating the base, the positive charge also depletes the emitter. The net effect of the oxide charge is still beneficial in the lightly-doped devices, because the increased recombination in the emitter is not sufficient to make up for the decreased recombination in the base. This is because the emitter doping is greater than the base
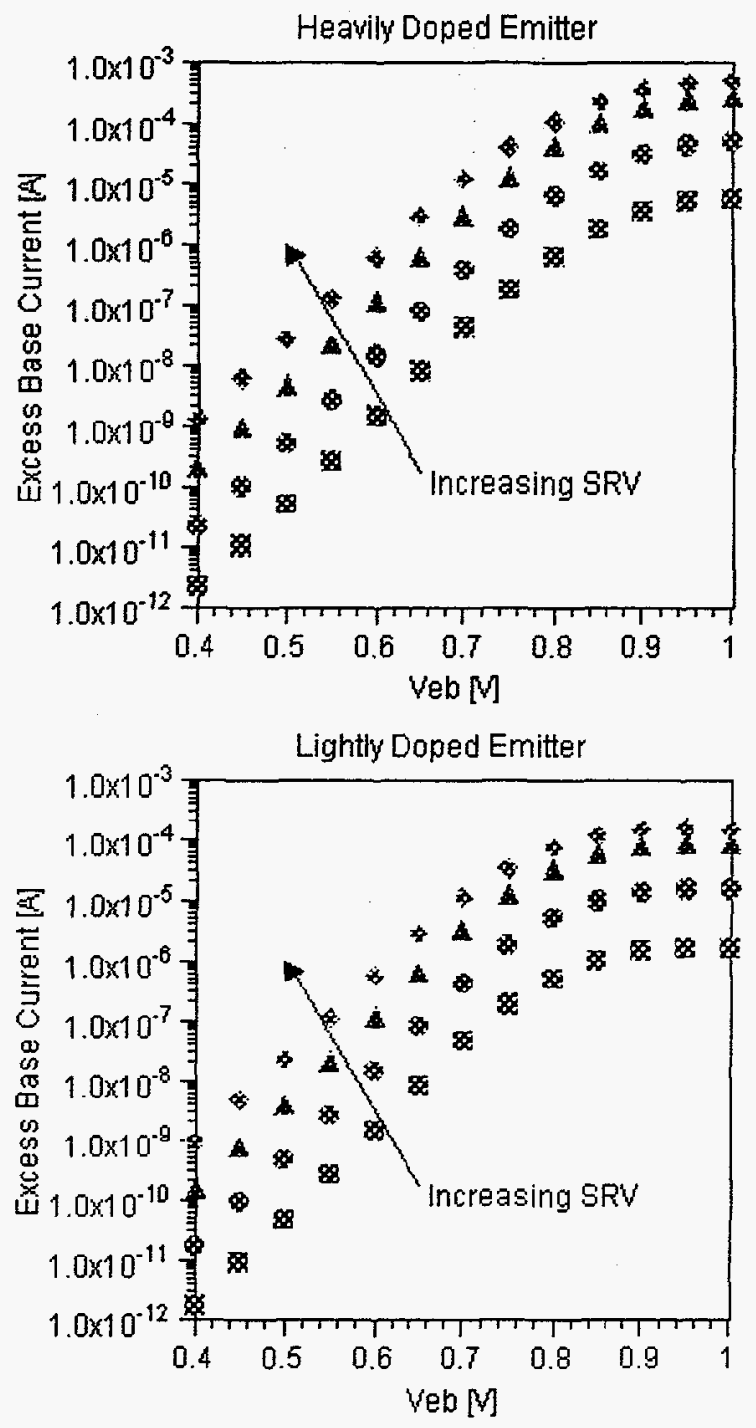

Figure 6. Excess base cument vs. emitter-base voltage at $\mathrm{N}_{\mathrm{ox}}=2 \times 10^{11}$ $\mathrm{cm}^{-2}$ plotted with increasing SRV

doping, even in the lightly-doped devices. These effects are illustrated in Fig. 8, using simulated structure files. The important areas to observe within the device are the $\mathrm{Si} / \mathrm{SiO}_{2}$ interface over the emitter/base depletion region and the surface of the neutral base. This figure shows the recombination rate as a function of position, with "hot" colors indicating regions of high recombination. In this figure, $N_{o x}=2 \times 10^{13} \mathrm{~cm}^{-2}$ and SRV $=1 \times 10^{5}$ $\mathrm{cm} / \mathrm{s}$. Note that in the lightly-doped device, the recombination in the emitter is much higher than it is in the heavily-doped device. This is the main reason responsible for the higher degradation in the lightly-doped device.

\section{CONCLUSIONS}

This work shows that the heavily and lightly doped emitters react similarly with ionizing radiation, but the details of the degradation are different. The VTC devices do show a similar dose rate effect to the Analog Devices ADRF LPNP 

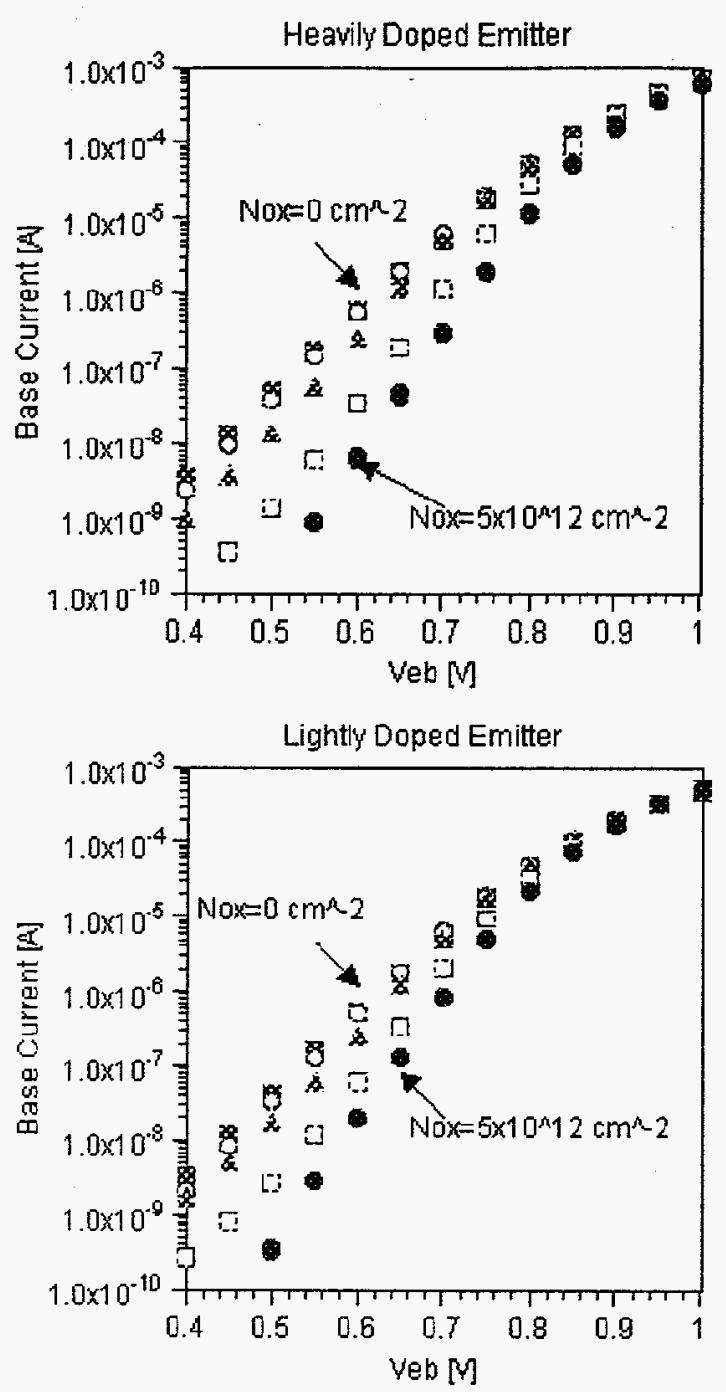

Figure 7. Base current vs. $V_{E B}$ for $S R V=1 \times 10^{5} \mathrm{~cm} / \mathrm{s}$
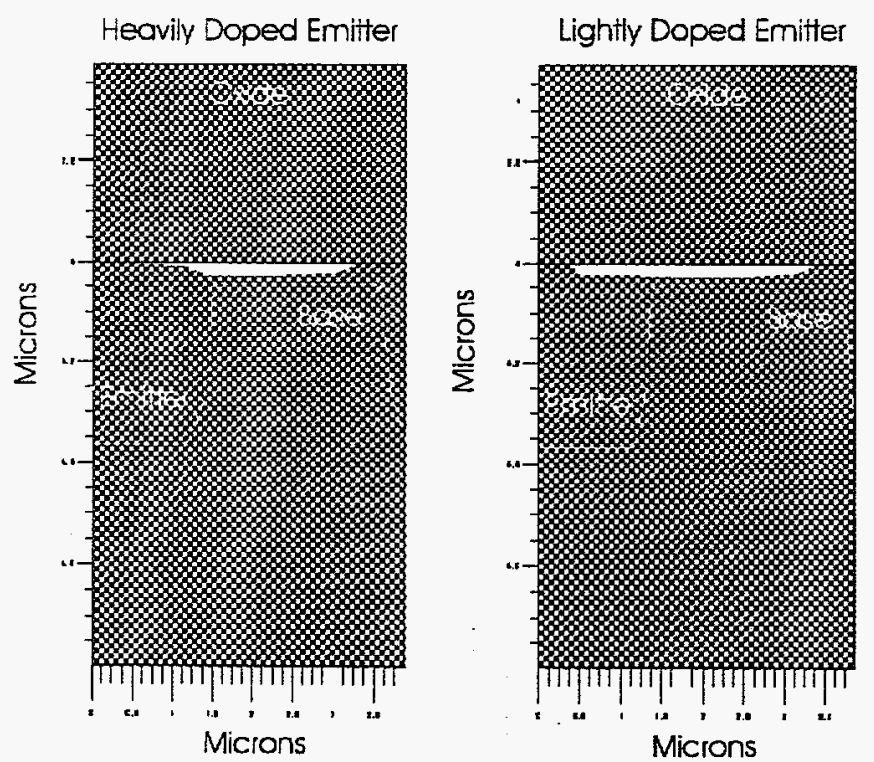

Figure 8. Comparison of recombination rates around the emitter base junction in both device types at $V_{\mathrm{EP}}=.65 \mathrm{~V}$ where the lowest dose rates show the most degradation. Also the devices with lightly-doped emitters degrade more rapidly with total dose because of the increased effect of radiation-induced charge on the emitter side of the depletion region. Both device types degrade rapidly as the surface recombination velocity increases, and the degradation is moderated by increased net positive oxide charge. Because of this similarity, it is probable that test methods developed using devices with heavily-doped emitters may apply to older technologies with lightly-doped emitters.

\section{REFERENCES}

[1] D.M. Schmidt, A. Wu, R.D. Schrimpf, D.M. Fleetwood, and R.L. Pease, "Modeling Ionizing Radiation Induced Gain Degradation of the Lateral PNP Bipolar Junction Transistor," IEEE Trans. Nucl. Sci., vol. 43, pp. 3032-3039, 1996.

[2] A.H. Johnston, G.M. Swift, and B.G. Rax, "Total Dose Effects in Conventional Bipolar Transistors and Linear Integrated Circuits," IEEE Trans. Nucl. Sci., vol. 41, pp. 2427 2436, 1994.

[3] S. McClure, R.L. Pease, W. Will, and G. Perry, "Dependence of Total Dose Response of Bipolar Linear Microcircuits on Applied Dose Rate," IEEE Trans. Nucl. Sci., vol. 41, pp. 2544-2549, 1994.

[4] J. Beaucour, T. Carriere, A. Gach, and P. Poirot, "Total Dose Effects on Negative Voltage Regulator," IEEE Trans. Nucl. Sci., vol. 41, pp. 2420-2426, 1994.

[5] D.M. Schmidt, D.M. Fleetwood, R.D. Schrimpf, R.L. Pease, R.J. Graves, G.H. Johnson, K.F. Galloway, and W.E. Combs, "Comparison of Ionizing-Radiation-Induced Gain Degradation in Lateral, Substrate, and Vertical PNP BJTs," IEEE Trans. Nucl. Sci., vol. 42, pp. 1541-1549, 1995.

[6] R.D. Schrimpf, R.J.Graves, D.M. Schmidt, D.M. Fleetwood, R.L. Pease, W.E. Combs, and M. DeLaus, "Hardness-Assurance Issues for Lateral PNP BJT's," IEEE Trans. Nucl. Sci., vol. 42, pp. 1641-1649, 1995.

[7] S.L. Kosier, R.D. Schrimpf, R.N. Nowlin, D.M. Fleetwood, M. DeLaus, R.L. Pease, W.E. Combs, A. Wei, and F. Chai, "Charge Separation for Bipolar Transistors," IEEE Trans. Nucl. Sci., vol. 40, no. 6, pp. 1276-1285, 1993.

[8] S.L. Kosier, A. Wei, R.D. Schrimpf, D.M. Fleetwood, and M. DeLaus, "Physically-Based Comparison of Hot-CarrierInduced and Ionizing-Radiation-Induced Degradation in BJTs," IEEE Trans. Electron Dev., vol. 42, pp. 436-444, 1995.

[9] S.L. Kosier, W.E. Combs, A. Wei, R.D. Schrimpf, D.M. Fleetwood, M. Delaus, and R.L. Pease, "Bounding the TotalDose Response of Modem Bipolar Transistors," IEEE Trans. Nucl. Sci., vol. 41, pp. 1864-1870, 1994.

[10] S.L. Kosier, R.D. Schrimpf, A. Wei, M. Delaus, D.M. Fleetwood, and W.E. Combs, "Effects of Oxide Charge and Surface Recombination Velocity on the Excess Base Current of BJT's," Proc. IEEE Bipolar/BiCMOS Circuits and Technology Meeting, vol. 41, pp. 211-214, 1994.

[11] R.D. Schrimpf, "Recent Advances in Understanding Total Dose Effects in Bipolar Transistors," IEEE Trans. Nucl. Sci., vol. 43, pp. 787-796, 1996. 